\title{
ALOCAÇÃO DE UNIDADES HIDRELÉTRICAS NO PROBLEMA DA PROGRAMAÇÃO DA OPERAÇÃO ENERGÉTICA UTILIZANDO RELAXAÇÃO LAGRANGEANA E LAGRANGEANO AUMENTADO
}

\author{
Rafael N. Rodrigues* \\ rafael@labplan.ufsc.br
}

\author{
Edson L. da Silva* \\ edsonelabplan.ufsc.br \\ * Departamento de Engenharia Elétrica, Universidade Federal de Santa Catarina \\ Florianópolis, SC, Brasil
}

\author{
Erlon C. Finardi* \\ erlonelabplan.ufsc.br
}

Floriatopolis, SC, Brasil

\begin{abstract}
The short-term operation planning problem aims to define the generation units should operate at minimum cost to fully supply the demand while meeting the system constraints over the study horizon. The problem is of a non-linear, integer-mixed, large scale optimization type, whose solution is a rather challenging task. This paper uses the Lagrangian Relaxation to decompose the original problem into simpler subproblems to be solved in sequence. In this decomposition, the subproblems are constructed taking advantage of their specific characteristics. One of the resulting subproblems of the relaxation scheme refers to the commitment of hydroelectric units. In order to solve this problem, an algorithm for exhaustive enumeration is proposed. This algorithm makes use of the Augmented Lagrangean method, which solves the resulting constrained nonlinear subproblems for each examined state. A Quasi-Newton method is used for the solution of the unconstrained subproblems resulting from the application of the Augmented Langrangean method. The proposed model is applied to two hydroelectric units of the Brazilian power system, demonstrating its consistency and practical feasibility.
\end{abstract}

Artigo submetido em 01/03/2004

1a. Revisão em 03/10/2005

2a. Revisão em 12/06/2006

Aceito sob recomendação do Editor Associado

Prof. Glauco Nery Taranto
KEYWORDS: Power System Operation Programming, Unit Dispatch, Lagrangian Relaxation, Augmented Lagrangian.

\section{RESUMO}

O problema da programação da operação energética visa definir quais unidades geradoras devem estar em operação para o atendimento à demanda e às demais restrições do sistema, ao longo do horizonte de estudo, de modo que o mínimo custo de operação seja encontrado. Matematicamente, trata-se de um problema não-linear, inteiro-misto e de grande porte, o que torna a sua solução uma tarefa desafiadora. Este artigo apresenta o uso da Relaxação Lagrangeana para decompor o problema da programação da operação em subproblemas menores e mais simples de serem solucionados. No esquema de decomposição utilizado, os subproblemas têm naturezas distintas e são construídos aproveitando-se as particularidades que cada um deles apresenta. Um dos subproblemas resultante do esquema de relaxação utilizado refere-se à alocação das unidades hidrelétricas. Para resolver esse subproblema, propõe-se um algoritmo de enumeração exaustiva do espaço de estados do problema. Cada combinação consiste na solução de problemas não-lineares restritos, resolvidos aqui por meio do método de Lagrangeano Aumentado. Tal método transforma cada problema restrito em uma série de subproblemas irrestritos, que por sua vez são solucionados por um algoritmo de Quase-Newton. O modelo compu- 
tacional desenvolvido é aplicado a duas usinas hidrelétricas do sistema brasileiro, demonstrando-se a sua consistência e viabilidade prática.

PALAVRAS-CHAVE: Programação da Operação Energética, Despacho de Unidades Hidrelétricas, Relaxação Lagrangeana, Lagrangeano Aumentado.

\section{INTRODUÇÃO}

O planejamento da operação constitui-se em um dos principais problemas da indústria de energia elétrica. Nessa tarefa, busca-se o atendimento da demanda ao menor custo possível por meio da otimização do uso dos recursos disponíveis. Usualmente o problema é subdividido em etapas, de modo que a modelagem do sistema tende a ser mais precisa à medida que se aproxima da operação em tempo real (Silva, 2001). Nesse contexto, a programação da operação corresponde à última dessas etapas.

A programação da operação tem por objetivo realizar a alocação das unidades geradoras ao longo do horizonte de estudo, isto é, definir quais unidades devem operar e os respectivos despachos, de modo a atender à demanda, os requisitos de reserva, limites de transmissão, e as diversas restrições operativas individuais das unidades e dos reservatórios do sistema.

Em face dos expressivos custos de operação, torna-se necessária uma representação precisa do comportamento físico e dos custos de produção das unidades geradoras. Isso, por sua vez, resulta em um modelo de otimização bastante complexo. Exemplificando, no caso de unidades termelétricas, a natureza termodinâmica impede que a potência de saída possa variar acima de valores específicos entre dois estágios consecutivos. Além disso, também relacionado com o processo termodinâmico, existem restrições do tipo minimum uptime and downtime ${ }^{1}$ que devem ser modeladas precisamente para evitar danos às unidades durante o processo de aquecimento e desaquecimento da caldeira de vapor.

No tocante ao uso dos recursos hídricos, restrições operativas relacionadas aos reservatórios e as unidades também devem ser consideradas. Essas restrições são de grande importância para sistema brasileiro, visto que a produção hidrelétrica corresponde $89 \%$ da produção total ${ }^{2}$. Portanto, pequenos ganhos percentuais de eficiência no uso dos recursos hídricos são significativos economicamente.

Dentre as diversas restrições do sistema hidrelétrico, merecem destaque àquelas ligadas com as unidades geradoras. A potência em uma unidade hidrelétrica depende do rendi-

\footnotetext{
${ }^{1}$ Restrições de número mínimo de estágios pelas quais uma unidade deve permanecer obrigatoriamente ligada ou desligada.

${ }^{2}$ No ano de 2004, a produção total de energia elétrica foi de 396.708 GWh.
}

mento do conjunto turbina-gerador, da altura de queda líquida e da vazão turbinada. A queda líquida é função nãolinear dos volumes armazenados no início e final de cada período e das vazões turbinada e vertida na usina. O rendimento tem seu comportamento descrito por meio de curvas de desempenho da unidade (curvas-colina), as quais usualmente informam também as chamadas zonas proibidas de geração. A operação em uma zona definida como proibida pode causar desde o desgaste excessivo da unidade ou mesmo desligamentos forçados para a sua manutenção e recuperação. Tais problemas são causados por cavitação ou por oscilações indesejáveis da potência de saída. A existência de tais zonas, representadas por limites específicos de potência, faz com que uma unidade possa ter vários estados operativos, aumentando a natureza combinatória do problema.

As características descritas acima fazem com que a solução do problema da programação da operação se constitua em uma tarefa desafiadora. O objetivo deste artigo é, inicialmente, apresentar uma modelagem que considera as características operativas das unidades geradoras de forma detalhada, com ênfase nas unidades hidrelétricas, em função de sua larga predominância no sistema brasileiro. Em seguida, faz-se o uso da técnica de Relaxação Lagrangeana (RL) (Bertsekas, 1999; Bonnans et al, 2002) para decompor o problema da programação em subproblemas menores. Esses subproblemas possuem naturezas distintas e são construídos aproveitando-se a estrutura particular que cada um deles apresenta.

Na seqüência, o foco deste artigo está nos subproblemas denominados Despacho de Unidades Geradoras Hidrelétricas. Conforme será mostrado, esse subproblema é de natureza contínua e não-linear. Para sua solução, o artigo apresenta o uso do método de Lagrangeano Aumentado (LA) (Martinez e Santos, 1998; Martinez et al, 2000), que utiliza, simultaneamente, os conceitos de dualidade e de penalidade quadrática.

A próxima seção é reservada para descrever a notação matemática utilizada. A Seção 3 refere-se à descrição dos aspectos de modelagem do problema. Na Seção 4 é apresentada a formulação do problema primal resultante da programação da operação energética. O procedimento de solução é foco principal da Seção 5. Na Seção 6 é apresentada a aplicação do algoritmo de programação não-linear necessário para definir a alocação das unidades geradoras hidrelétricas. Por fim, na última seção do trabalho são apresentadas as principais conclusões.

\section{NOTAÇÃO}

A seguinte notação é utilizada neste trabalho:

$G$ constante com valor de $9,8110^{-3}\left(\mathrm{~kg} / \mathrm{m}^{2} \mathrm{~s}^{2}\right)$; 
$T$ número total de estágios da programação, em horas;

$t$ índice dos estágios da programação, onde $t=1, T$;

$R$ número total de reservatórios do sistema;

$r$ índice dos reservatórios do sistema, onde $r=1, R$;

$J(r)$ número de unidades no reservatório $r$;

$j$ índice de unidades hidrelétricas, onde $j=1, J(r)$;

$\eta_{j r t}$ rendimento da unidade $j$, reservatório $r$ e estágio $t$;

$\rho_{1 j}, \ldots, \rho_{5 j}$ são coeficientes de eficiência de cada unidade;

$h l_{j r t}$ queda líquida na unidade $j$, reservatório $r$ e estágio $t(m)$

$q_{j r t}$ vazão turbinada na unidade $j$, reservatório $r$ e estágio $t\left(m^{3} / s\right)$;

$v_{r t}$ volume no reservatório $r$, início do estágio $t\left(h \mathrm{~m}^{3}\right)$;

$v_{r}^{\min , \max }$ limites de volumes do reservatório $r\left(h \mathrm{~m}^{3}\right)$;

$Q_{r t}$ vazão turbinada no reservatório $r$, estágio $t\left(\mathrm{~m}^{3} / \mathrm{s}\right)$;

$s_{r t}$ vazão vertida no reservatório $r$, estágio $t\left(\mathrm{~m}^{3} / \mathrm{s}\right)$;

$Q a_{r t}$ variável duplicada de $Q_{r t}$;

$s a_{r t}$ variável duplicada de $s_{r t}$;

$f c m_{r t}($.$) cota montante da usina r$, estágio $t(m)$;

$f c j_{r t}($.$) cota jusante da usina r$, estágio $t(m)$;

$p l_{j r t}$ perdas hidráulicas na unidade $j$, reservatório $r$ e estágio $t(m)$;

$k p_{r}$ constante de perdas hidráulicas associada ao conduto forçado comum do reservatório $r\left(s^{2} / m^{5}\right)$;

$k p_{j r}$ constante de perdas hidráulicas associada ao conduto forçado da unidade $j$ e reservatório $r\left(s^{2} / m^{5}\right)$;

$p h_{j r t}$ potência de saída da unidade $j$, reservatório $r$ e estágio $t$ (MW);

$p h_{j k r t}^{\min , \max }$ limites de potência da unidade $j$, reservatório $r$ e estágio $t$, com a mesma operando na zona $k(\mathrm{MW})$;

$G h_{r t}$ variável artificial que representa o somatório das potências de saída das unidades geradoras da usina $r$ no estágio $t$;

$\Phi_{j r}$ total de zonas permitidas de operação da unidade $j$ do reservatório $r$;

$k$ índice das zonas das unidades hidrelétricas, onde $k=$ $1, \Phi_{j r} ;$ $z_{j k r t}$ variável binária que indica se a unidade $j$, reservatório $r$ está ligada (igual a 1) ou desligada (igual a 0 ) na zona $k$ durante o estágio $t$;

$R_{+}^{(r)}$ conjunto de reservatórios imediatamente a montante de $r$

$\tau_{m r}$ tempo de viagem d'água entre os reservatórios $m$ e $r(h)$

$y_{r t}$ vazão lateral afluente ao reservatório $r$, estágio $t\left(m^{3} / s\right)$;

$r h_{r t}$ reserva da usina hidrelétrica $r$, estágio de $t(\mathrm{MW})$;

$\alpha$ custo futuro de operação esperado no final do horizonte da programação $(\mathrm{R} \$)$;

$P$ total de segmentos da função linear por partes $\alpha$;

$p$ índice associado ao segmento linear da função $\alpha$, com $p=1, P$;

$\pi_{r}^{(p)}$ constante do reservatório $r$ e aproximação $p$ da função linear por partes que representa $\alpha\left(\mathrm{R} \$ / h m^{3}\right)$;

$\alpha_{0}^{(p)}$ constante referente à aproximação $p$ de $\alpha$;

$I$ total de usinas termelétricas do sistema;

$i$ índice das unidades termelétricas, onde $i=1, I$;

$p t_{i t}$ potência de saída da unidade $i$, estágio $t$ (MW);

$p t a_{i t}$ variável duplicada de $p t_{i t}$;

$c_{i t}($.$) custo de operação da unidade i$, estágio $t(\mathrm{R} \$)$;

$\omega$ conjunto de restrições das termelétricas;

$E$ total de subsistemas do sistema hidrotérmico;

$e$ índice associado aos subsistemas, onde $e=1, E$;

$I_{e}$ índices das usinas termelétricas do subsistema $e$;

$R_{e}$ índices dos reservatórios do subsistema $e$;

$\Omega_{e}$ subsistemas interligados ao subsistema $e$;

Int $t_{\text {elt }}$ intercâmbio do subsistema $e$ para $l$, estágio $t$ (MW);

$D_{\text {et }}$ demanda do subsistema $e$, estágio $t$ (MW);

Int $t_{\text {elt }}^{\max }$ limites de intercâmbio do subsistema $e$ para o subsistema $l$, estágio $t$ (MW);

$u_{i t}$ variável binária que indica se a unidade $i$ está ligada (igual a 1) ou desligada (igual a 0), no estágio $t$;

$x_{i t}$ tempo total que a unidade $i$ está ligada ou desligada, referente ao estágio $t(h)$; 
$c_{v}$ constante que transforma vazão $\left(\mathrm{m}^{3} / \mathrm{s}\right)$ em volume $\left(h \mathrm{~m}^{3}\right)$, num período de tempo igual a uma hora;

$\ell_{A}$ função Lagrangeano Aumentado;

$\nabla \ell_{A}$ gradiente da função Langrageano Aumentado;

$c_{m}(x)$ restrições do problema a ser resolvido por Lagrangeano Aumentado;

$m$ índice de restrições do problema;

$\lambda_{m}$ multiplicador de Lagrange associados às restrições $c_{m}(x)$;

$\mu$ parâmetro de penalidade da função Lagrangeano Aumentado;

it índice de iterações de processo iterativo;

$\beta_{j}$ variáveis de folga utilizadas nas restrições de limites de potência;

$\beta_{R}$ variável de folga utilizada na restrição de reserva energética de uma determinada usina;

$\Theta_{H T}$ problema dual de programação da operação;

$\Theta_{A U T}$ subproblema de alocação de unidades termelétricas;

$\Theta_{D}$ subproblema de atendimento à demanda;

$\Theta_{H}$ subproblema hidrelétrico;

$\Theta_{S H}$ subproblema hidráulico;

$\Theta_{A U H}$ subproblema de alocação de unidades hidrelétricas;

$\Theta_{D U H}^{t}$ subproblema de despacho de unidades hidrelétricas para cada estágio $t$;

$\lambda p t_{i t}$ multiplicadores de Lagrange associados às restrições de duplicação das variáveis $p t_{i t}$;

$\lambda d_{r t}$ multiplicadores de lagrange associados às restrições de duplicação das variáveis $p h_{j r t}$;

$\lambda q_{r t}$ multiplicadores de Lagrange associados às restrições de duplicação das variáveis $Q_{r t}$;

$\lambda s_{r t}$ multiplicadores de Lagrange associados às restrições de duplicação das variáveis $s_{r t}$;

ng número de unidades geradoras no problema de despacho de unidades hidrelétricas.

\section{MODELAGEM DAS UNIDADES GERA- DORAS}

A função de produção de uma unidade hidrelétrica é dada por:

$$
p h_{j r t}=G \eta_{j r t} h l_{j r t} q_{j r t}
$$

sendo a altura de queda líquida definida por:

$$
\begin{array}{r}
h l_{j r t}=f c m_{r t}\left(v_{r t}, v_{r, t+1}\right) f c j_{r t}\left(Q_{r t}+s_{r t}\right)- \\
p l_{j r t}\left(Q_{j r t}, q_{j r t}\right)
\end{array}
$$

A função $f_{c m}\left(m_{r t}(\cdot)\right.$ é descrita, usualmente, por um polinômio de quarta ordem. Todavia, em estudos de curto prazo ${ }^{3}$, conforme é o caso da programação da operação, a variação da cota de montante dos reservatórios não é significativa. Assim, neste trabalho o valor de $f \mathrm{~cm}_{r t}(\cdot)$ é considerado constante ao longo de todo o horizonte de estudo. Por sua vez, a função $f c j_{r t}(\cdot)$, descrita por um polinômio de mesma ordem, é uma função da vazão turbinada na usina e do vertimento durante o estágio de tempo em consideração. O último termo de (3.2), $p l_{j r t}($.), descreve as perdas hidráulicas no conduto forçado de cada unidade devido ao atrito associado ao movimento da água entre as cotas de montante e jusante. Essas perdas são modeladas como sendo proporcionais ao quadrado da vazão turbinada em cada unidade geradora e da vazão turbinada total da usina (Cicogna et al, 2003):

$$
p l_{j r t}=k p_{r} Q_{r t}^{2}+k p_{j r} q_{j r t}^{2}
$$

Para o horizonte de programação da operação considerado, uma representação realista do rendimento da turbina e do gerador é importante para proporcionar um despacho otimizado. Além disso, as unidades geradoras têm zonas proibidas para a geração. Essas zonas proibidas, bem como o complexo inter-relacionamento entre as variáveis, são usualmente representadas na curva-colina, tal como é ilustrado na Figura 3.1. Nessa figura, por exemplo, observam-se os rendimentos de uma unidade, representados por meio de curvas de nível, limites da vazão turbinada, altura de queda líquida e potência de saída da unidade. É possível observar ainda que essa unidade possui apenas uma zona proibida de geração, compreendida entre 0 e $80 \mathrm{MW}$.

$\mathrm{O}$ rendimento, $\eta_{j r t}$, depende da queda líquida e da vazão turbinada. Neste trabalho, $\eta_{j r t}$ é representado pela seguinte função:

$$
\begin{aligned}
\eta_{j r t}=\rho_{0 j r}+\rho_{1 j r} h l_{j r t}+\rho_{2 j r} q_{j r t}+ \\
\rho_{3 j r} h l_{j r t} q_{j r t}+\rho_{4 j r} h l_{j r t}^{2}+\rho_{5 j r} q_{j r t}^{2}
\end{aligned}
$$

Os coeficientes de (3.4) podem ser estimados por meio de técnicas de Regressão Não-Linear Multivariável (Wonnacott, 1972).

\footnotetext{
${ }^{3}$ Em geral o horizonte da programação varia de 24 a 168 horas.
} 
Portanto, com base nessas considerações, a função que descreve a potência de saída tem a seguinte estrutura:

$$
p h_{j r t}\left(q_{j r t}, Q_{r t}, s_{r t}\right)=f\left(\left[q_{j r t}\right]^{7},\left[Q_{r t}\right]^{12},\left[s_{r t}\right]^{12}\right)
$$

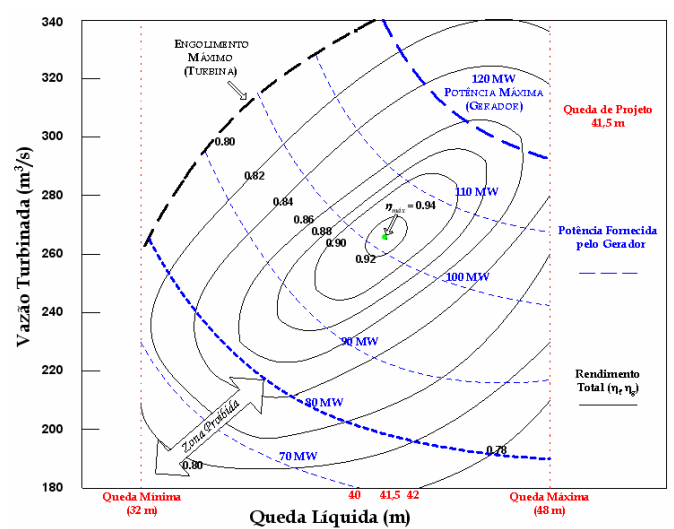

Figura 3.1: Curva-Colina de Uma Unidade Hidrelétrica.

\section{FORMULAÇÃO DO PROBLEMA}

A formulação do problema de programação da operação é representada, matematicamente, da seguinte forma:

$$
\min \sum_{t=1}^{T} \sum_{i=1}^{I} c_{i t}\left(p t_{i t}, x_{i, t-1}, u_{i t}, u_{i, t-1}\right)+\alpha
$$

s.a:

$$
\begin{gathered}
\sum_{i \in I_{e}} p t_{i t}+\sum_{r \in R_{e}}\left(\sum_{j=1}^{J(r)} p h_{j r t}\left(q_{j r t}, Q_{r t}, s_{r t}\right)\right)+ \\
\sum_{l \in \Omega_{e}}\left(\text { Int }_{l e t}-I_{n t_{e l t}}\right)=D_{e t} \\
-I n t_{l e t}^{\max _{1}} \leq I n t_{l e t} \leq I n t_{\text {let }}^{\max _{2}} \\
v_{r, t+1}-v_{r t}+c_{v}\left[Q_{r t}+s_{r t}-\right. \\
\left.\sum_{m \in R_{+}^{(r)}}\left(Q_{m, t-\tau_{m r}}+s_{m, t-\tau_{m r}}\right)-y_{r t}\right]=0
\end{gathered}
$$

$$
\begin{aligned}
& \alpha+\sum_{e=1}^{E} \sum_{r \in R_{e}} \pi_{r}^{(p)} v_{r, T+1} \geq \alpha_{0}^{(p)} \\
& v_{r}^{\text {min }} \leq v_{r, t+1} \leq v_{r}^{\max } \\
& s_{r t} \geq 0 \\
& Q_{r t}=\sum_{j=1}^{J(r)} q_{j r t} \\
& 0 \leq q_{j r t} \leq q_{j r t}^{\max } \\
& \sum_{k=1}^{\Phi_{j r}} p h_{j k r t}^{\min } z_{j k r t} \leq p h_{j r t}\left(q_{j r t}, Q_{r t}, s_{r t}\right) \leq \\
& \sum_{k=1}^{\Phi_{j r}} p h_{j k r t}^{\max } z_{j k r t} \\
& z_{j k r t} \in\{0,1\} \\
& \sum_{k=1}^{\Phi_{j r}} z_{j k r t} \leq 1
\end{aligned}
$$

$$
\sum_{j=1}^{J(r)}\left(\sum_{k=1}^{\Phi_{j r}} p h_{j 1 r t}^{\max } z_{j k r t}-p h_{j r t}\left(q_{j r t}, Q_{r t}, s_{r t}\right)\right) \geq r h_{r t}
$$$$
p t_{i t}, x_{i t}, u_{i t} \in \omega
$$

$$
\begin{array}{cccc}
e=1, E & i=1, I & r=1, R & p=1, P \\
t=1, T & j=1, J(r) & k=1, \Phi_{j r} & l \in \Omega_{e}
\end{array}
$$

A função objetivo (4.1) visa a minimização do custo de produção no presente, associado ao uso das termelétricas, e também do custo futuro de operação, sendo este uma função do nível de armazenamento dos reservatórios ao final do horizonte de estudo. Em linhas gerais, quanto maior o nível de armazenamento ao final do horizonte de estudo, menor será o valor do custo de operação esperado para o futuro. Esta função é matematicamente representada por uma função linear por partes, sendo obtida por modelos de planejamento da operação de mais longo prazo (Silva e Finardi, 2003).

Sendo o foco deste trabalho o sistema hidrelétrico, a modelagem das usinas termelétricas é descrita de maneira simplificada. Assim, o custo de operação $c_{i t}(\cdot)$ considera os custos de produção de energia e de partida. Por sua vez, a Restrição (4.13) engloba todas as restrições associadas às unidades termelétricas, tais como número mínimo de estágios de tempo que devem estar ligadas ou desligadas, variações de potência entre dois intervalos de tempo, limites de operacionais e de reserva. 
O conjunto de restrições (4.2) refere-se ao atendimento da demanda em cada subsistema durante o estágio $t$. As restrições (4.3) definem os limites do intercâmbio de energia entre os subsistemas durante o estágio $t$. Já (4.4)-(4.12) dependem das variáveis de natureza hidrelétrica, isto é, $z_{j k r t}, q_{j r t}, Q_{r t}$, $s_{r t}$ e $v_{r t}$. As restrições (4.10) representam as zonas proibidas de geração. Ainda, a Restrição (4.2) acopla as variáveis de geração e de intercâmbio, ou seja, $p h_{j r t}$, ptit, Int let e Int $t_{\text {elt }}$. Esse grau de acoplamento torna a solução do problema uma tarefa difícil de ser realizada. A próxima seção ilustra a estratégia de solução empregada para resolver (4.1)-(4.13).

\section{DECOMPOSIÇÃO DO PROBLEMA}

O Problema Primal (4.1)-(4.13) é de natureza não-linear, inteiro-misto, e de grande porte. Esse problema pode ser resolvido por meio da relaxação de algumas restrições e assim requerendo a construção de um problema auxiliar, denominado de problema dual. Maximizando tal função e ajustando soluções primais obtidas a partir dessa maximização, podese então encontrar soluções primais viáveis de (4.1)-(4.13) (Geoffrion, 1971; Urruty e Lemarechal, 1993).

Encontrada uma solução dual, em problemas não-convexos, como é o caso, os valores da função objetivo do problema original e da função dual podem não ser iguais. Nesse caso, o valor da função dual é sempre menor ou igual ao valor ótimo do Problema Primal (4.1)-(4.13) e a diferença entre tais valores é chamada de gap de dualidade (Geoffrion, 1971; Urruty e Lemaréchal, 1993). Todavia, para que essas soluções sejam de boa qualidade, é crucial que o gap de dualidade seja suficientemente pequeno.

Para garantir que o gap seja o menor possível, este trabalho faz uso de uma técnica de decomposição conhecida como splitting methods (Douglas e Rachford, 1956), os quais têm sido utilizados em programação estocástica (Ruszczynski, 1997) e em determinados problemas de natureza combinatória (Batut e Renaud, 1992; Lemarechal et al, 1996; Renaud, 1993). A idéia consiste em duplicar artificialmente as variáveis primais de interesse para, posteriormente, encontrar uma decomposição adequada por meio da dualização das restrições artificialmente impostas e resultantes dessa duplicação. Essa estratégia tem sido utilizada com sucesso no problema de alocação de unidades termelétricas (Batut e Renaud, 1992; Lemaréchal et al, 1996; Renaud, 1993) e será utilizada neste trabalho.

Na seqüência descreve-se o esquema de decomposição usado neste trabalho para resolver o Problema (4.1)-(4.13). Inicial- mente, esse problema é rescrito da seguinte maneira:

$$
\begin{aligned}
& \min =\sum_{t=1}^{T} \sum_{i=1}^{I} c_{i t}\left(p t_{i t}, x_{i, t-1}, u_{i t}, u_{i, t-1}\right)+\alpha \\
& \text { s.a: } \\
& \text { (4.2)-(4.13) } \\
& p t a_{i t}=p t_{i t} \\
& G H_{r t}=\sum_{j=1}^{J(r)} p h_{j r t}\left(q_{j r t}, Q_{r t}, s_{r t}\right)
\end{aligned}
$$

O próximo passo consiste em substituir as variáveis $p t a_{i t} \mathrm{e}$ $G H_{r t}$ nas restrições de atendimento a demanda. As demais restrições permanecem intactas:

$$
\begin{aligned}
& \min =\sum_{t=1}^{T} \sum_{i=1}^{I}\left[c_{i t}\left(p t_{i t}\right)+s t_{i t}\left(x_{i, t-1}\right) u_{i, t}\left(1-u_{i, t-1}\right)\right]+\alpha \\
& \text { s.a: } \\
& \quad \sum_{i \in I_{e}} p t a_{i t}+\sum_{r \in R_{e}} G H_{t}+\sum_{l \in \Omega_{e}}\left(\text { Int }_{\text {let }}-\text { Int }_{\text {elt }}\right)=D_{\text {et }} \\
& \quad(4.3)-(4.17) \\
& p t a_{i t}=p t_{i t} \\
& G H_{r t}=\sum_{j=1}^{J(r)} p h_{j r t}\left(q_{j r t}, Q_{r t}, s_{r t}\right)
\end{aligned}
$$

$\mathrm{Na}$ etapa seguinte, as restrições artificiais impostas em (5.1) são dualizadas, sendo $\lambda p t_{i t}$ e $\lambda d_{r t}$ os multiplicadores de Lagrange associados à tais restrições. Procedendo desta forma, tem-se que o problema dual resultante, $D_{H T}$, pode ser resolvido por $(I+T+1)$ subproblemas desacoplados entre si:

$$
\begin{aligned}
& \left(D_{H T}\right)=\max \Theta\left(\lambda p t_{i t}, \lambda d_{r t}\right)= \\
& \sum_{i=1}^{T} \Theta_{A U T}^{i}\left(\lambda p t_{i t}\right)+\sum_{t=1}^{T} \Theta_{D}^{t}\left(\lambda p t_{i t}, \lambda d_{r t}\right)+\Theta_{H}\left(\lambda d_{r t}\right)
\end{aligned}
$$

onde

$\Theta_{A U T}^{i}()=.\min \sum_{t=1}^{T} c_{i t}\left(p t_{i t}, x_{i, t-1}, u_{i t}, u_{i, t-1}\right)-\lambda p t_{i t} p t_{i t}$

s.a: (4.13)

$$
\begin{gathered}
\Theta_{D}^{t}(.)=\min \left\{\sum_{i=1}^{I} \lambda p t_{i t} p t a_{i t}+\sum_{r=1}^{R} \lambda d_{r t} G H_{r t}\right\} \\
\text { s.a.: } \sum_{i \in I_{e}} p t a_{i t}+\sum_{r \in R_{e}} G H_{t}+\sum_{l \in \Omega_{e}}\left(\text { Int }_{\text {let }}-\text { Int }_{\text {elt }}\right)=D_{\text {et }}
\end{gathered}
$$

(4.3) e ( $t$ fixo) 
$\Theta_{H}()=.\min \alpha-\sum_{t=1}^{T} \sum_{r=1}^{R} \lambda d_{r t}\left(\sum_{j=1}^{J} p h_{j r t}\left(q_{j r t}, Q_{r t}, s_{r t}\right)\right)$

s.a: (4.10)-(4.12)

O Subproblema (5.4) representa as termelétricas., comumente resolvido por técnicas de Programação Dinâmica (Bellman, 1957). No tocante ao subproblema de atendimento à demanda, definido por (5.5), o mesmo é constituído apenas por variáveis contínuas e, devido à representação simplificada do sistema de transmissão, esse subproblema é de natureza linear. Outra observação importante é o fato de que esse subproblema é desacoplado no tempo, podendo ser, portanto, resolvido a cada estágio de tempo por meio de qualquer algoritmo de programação linear. Por fim, pode-se notar que o Subproblema (5.6) está relacionado com variáveis do sistema hidrelétrico. No caso brasileiro, devido a predominância dos recursos hídricos, esse subproblema é ainda de grande porte, não-linear e inteiro-misto. Isso significa que novas decomposições são necessárias para resolver o problema dual (5.3). A solução do subproblema hidrelétrico é tratada na próxima seção.

\subsection{Solução do Subproblema Hidrelétrico}

As restrições (4.4)-(4.7) são compostas por variáveis que dizem respeito à modelagem dos reservatórios, isto é, $Q_{r t}$, $s_{r t}$ e $v_{r, t+1} \mathrm{e} \alpha$. Já o conjunto de restrições definido por (4.8)-(4.12) tem, além de $Q_{r t}$ e $s_{r t}$, variáveis associadas estritamente às unidades geradoras, no caso $q_{j r t}$ e $z_{r k j t}$. Pode-se notar ainda que as características das restrições envolvidas nesses dois conjuntos são de naturezas distintas. No primeiro, existem apenas restrições lineares enquanto no segundo estão presentes restrições não-lineares e inteiramistas. Ainda, no subconjunto de restrições definido por (4.4)-(4.8) existe um acoplamento temporal e espacial entre os reservatórios. Já o subconjunto de restrições restante se caracteriza pela inexistência desses acoplamentos. Note-se então que a decomposição proposta, e apresentada a seguir, faz com que as características citadas acima sejam exploradas convenientemente e tratadas de forma independente.

Para realizar a decomposição proposta, devem-se identificar as variáveis que são comuns aos dois conjuntos citados. O acoplamento entre esses dois conjuntos de restrições é feito pelas variáveis $Q_{r t}$ e $s_{r t}$. Assim, pode-se fazer uso novamente da estratégia de duplicação de variáveis, adicionando as seguintes restrições artificiais:

$$
\begin{aligned}
& \min \alpha-\sum_{t=1}^{T} \sum_{r=1}^{R} \lambda d_{r t} \sum_{j=1}^{J(r)} p h_{j r t}\left(q_{j r t}, Q_{r t}, s_{r t}\right) \\
& \text { s.a: } \\
& Q a_{r t}=Q_{r t} \\
& s a_{r t}=s_{r t} \\
& \text { (4.4)-(4.12) }
\end{aligned}
$$

Na reconstrução de (5.1.1), as variáveis artificiais $Q a_{r t}$ e $s a_{r t}$ substituem as variáveis $Q_{r t}$ e $s_{r t}$ nas restrições (4.4):

$$
\Theta_{H}(.)=\min \alpha-\sum_{t=1}^{T} \sum_{r=1}^{R} \lambda d_{r t} \sum_{j=1}^{J} p h_{j r t}\left(q_{j r t}, Q_{r t}, s_{r t}\right)
$$

s.a:

$$
\begin{aligned}
& v_{r, t+1}-v_{r t}+c_{v}\left[Q a_{r t}+s a_{r t}-\right. \\
& \left.\sum_{m \in R_{+}^{(r)}}\left(Q_{m, t-\tau_{m r}}+s_{m, t-\tau_{m r}}\right)-y_{r t}\right]=0
\end{aligned}
$$

$$
\begin{aligned}
& Q a_{r t}=Q_{r t} \\
& s a_{r t}=s_{r t} \\
& (4.5)-(4.12)
\end{aligned}
$$

A etapa seguinte consiste em relaxar as restrições associadas às variáveis artificiais (5.1.4) e (5.1.5), onde $\lambda q_{r t}$ e $\lambda s_{r t}$ são os respectivos multiplicadores de Lagrange dessas restrições. Nesse sentido, tem-se:

$$
\begin{aligned}
& \max \Theta_{H}\left(\lambda d_{r t}, \lambda q_{r t}, \lambda s_{r t}\right)= \\
& \min \alpha-\sum_{t=1}^{T} \sum_{r=1}^{R} \lambda d_{r t} \sum_{j=1}^{J(r)} p h_{j r t}\left(q_{j r t}, Q_{r t}, s_{r t}\right)+ \\
& \sum_{t=1}^{T} \sum_{r=1}^{R}\left[\lambda q_{r t}\left(Q_{r t}-Q a_{r t}\right)+\lambda s_{r t}\left(s_{r t}-s a_{r t}\right)\right] \\
& \text { s.a: }
\end{aligned}
$$$$
\text { (5.1.3), (4.5)-(4.12) }
$$

A função dual acima pode ser avaliada por meio da resolução de dois novos conjuntos de subproblemas. O primeiro surge agrupando-se os termos da função objetivo e as restrições que dependem exclusivamente das variáveis de reservatórios 


$$
\begin{aligned}
& \left(\alpha, v_{r, t+1}, Q a_{r t}, s a_{r t}\right): \\
& \Theta_{S H}\left(\lambda q_{r t}, \lambda s_{r t}\right)=\min \alpha-\sum_{t=1}^{T} \sum_{r=1}^{R}\left[\lambda q_{r t} Q a_{r t}+\lambda s_{r t} s a_{r t}\right] \\
& \text { s.a }:(5.1 .3),(4.5)-(4.12)
\end{aligned}
$$

Este subproblema é de natureza linear, acoplado no tempo e no espaço, sendo denominado aqui de subproblema hidráulico, podendo ser resolvido facilmente.

O segundo conjunto de subproblemas refere-se ao agrupamento das restrições que possuem as variáveis $q_{j r t}, Q_{r t}$, $s_{r t}$ e $z_{j k r t}$ :

$$
\begin{aligned}
& \Theta_{A U H}\left(\lambda d_{r t}, \lambda q_{r t}, \lambda s_{r t}\right)= \\
& \min -\sum_{t=1}^{T} \sum_{r=1}^{R} \lambda d_{r t} \sum_{j=1}^{J(r)} p h_{j r t}\left(q_{j r t}, Q_{r t}, s_{r t}\right)+ \\
& \sum_{t=1}^{T} \sum_{r=1}^{R}\left[\lambda q_{r t} Q_{r t}+\lambda s_{r t} s_{r t}\right]
\end{aligned}
$$

s.a: (4.8)-(4.12)

O subproblema acima é chamado de subproblema de alocação de unidades hidrelétricas, o qual é resolvido sem acoplamentos espaciais e temporais entre reservatórios, isto é:

$\Theta_{A U H}\left(\lambda d_{r t}, \lambda q_{r t}, \lambda s_{r t}\right)=\sum_{t=1}^{T} \sum_{r=1}^{R} \Theta_{A U H}^{r t}\left(\lambda d_{r t}, \lambda q_{r t}, \lambda s_{r t}\right)$

onde:

$$
\begin{array}{r}
\Theta_{A U H}^{r t}(.)=\min -\lambda d_{r t}\left(\sum_{j=1}^{J} p h_{j r t}\left(q_{j r t}, Q_{r t}, s_{r t}\right)\right)+ \\
\lambda q_{r t} Q_{r t}+\lambda s_{r t} s_{r t}
\end{array}
$$

s.a: (4.8)-(4.12), com $r$ e $t$ fixos.

Esse problema merece especial atenção, pois além das nãolinearidades envolvidas, o número de unidades e suas respectivas zonas operativas definem o tamanho do espaço de estados para análise, cuja avaliação computacional pode ser muito onerosa. Uma usina com $J(r)$ unidades diferentes, cada uma delas possuindo $k$ zonas permitidas para operação, têm um total de $(k+1)^{J(r)}$ combinações. Todavia, em geral, as usinas hidrelétricas do sistema brasileiro possuem unidades idênticas. Considerando o caso de unidades com uma única zona operativa (que também é o caso mais comum), o total de combinações decresce de $2^{J(r)}$ para $J(r)+1$. Portanto, neste caso, uma boa estratégia de solução para (5.1.10) consiste em investigar todo o espaço de estados por meio de uma enumeração exaustiva das combinações.
Usinas hidrelétricas com um grande número de unidades geradoras e/ou zonas operativas tornam o uso da enumeração exaustiva ineficiente, visto o grande número de combinações possíveis e, por conseqüência, do elevado esforço computacional. Para sobrepujar essa dificuldade, uma alternativa que pode ser adotada é, novamente, o uso da Relaxação Lagrangeana. Assim, uma nova decomposição pode ser realizada, dualizando aquelas restrições com presença de variáveis inteiras, (4.10)-(4.12) (Finardi et al, 2005).

Ainda no tocante ao problema (5.1.10), utilizando a enumeração exaustiva, o mesmo pode ser resolvido pela investigação das possíveis combinações de alocação de unidades, requerendo, portanto, solução de problemas não-lineares contínuos, chamados de despacho de unidades geradoras hidrelétricas:

$$
\begin{array}{r}
\Theta_{D U H}^{r t}(.)=\min -\lambda d_{r t}\left(\sum_{j=1}^{n g} p h_{j r t}\left(q_{j r t}, Q_{r t}, s_{r t}\right)\right)+ \\
\lambda q_{r t} Q_{r t}+\lambda s_{r t} s_{r t}
\end{array}
$$

$$
\begin{aligned}
& Q_{r t}=\sum_{j=1}^{n g} q_{j r t} \\
& p h_{j k r t}^{\min } \leq p h_{j r t}\left(q_{j r t}, Q_{r t}, s_{r t}\right) \leq p h_{j k r t}^{\max } j=1, n g \\
& \sum_{j=1}^{n g}\left[p h_{j 1 r t}^{\max }-p h_{j r t}\left(q_{j r t}, Q_{r t}, s_{r t}\right)\right] \geq r h_{r t}
\end{aligned}
$$

Assim, na seqüência deste artigo apresenta-se um algoritmo de programação não-linear que resolve os problemas de despacho de unidades hidrelétricas (5.1.11). Dessa forma, a próxima seção aborda a aplicação do método de LA como ferramenta de implementação desse algoritmo e a sua aplicação no problema de despacho de unidades geradoras hidrelétricas.

\subsection{Solução do Subproblema de Despa- cho de Unidades Geradoras Hidrelé- tricas}

Conforme visto na Seção 5.1.1, o Subproblema de Alocação de Unidades Hidrelétricas requer um algoritmo que solucione cada um dos problemas não-lineares contínuos. Para tanto, este artigo utiliza o método do LA, o qual é baseado nos conceitos de penalidade quadrática e de dualidade (Nocedal e Wright, 1999). Esse algoritmo transforma o problema com restrições em uma série de subproblemas irrestritos por meio da incorporação de penalidades às violações das restrições do problema. Os subproblemas irrestritos são aqui resolvidos pelo método de Quase-Newton (Martinez, 1998; Martinez, 2000). 
Considerando o problema de programação não-linear:

$$
\begin{aligned}
& \min f(x) \\
& \text { s.a: } \\
& c_{m}(x)=0 \\
& x^{\min } \leq x \leq x^{\max }
\end{aligned} \quad m=1, M
$$

a função Lagrangeano Aumentado possui a seguinte forma:

$$
\ell_{A}=f(x)+\sum_{m=1}^{M}\left(\lambda_{m} c_{m}(x)+\frac{1}{2 \mu}\left[c_{m}(x)\right]^{2}\right)
$$

O método do LA, a cada iteração, resolve um subproblema irrestrito, condicionado pelos valores de $\lambda_{m}$ e $\mu$.. O parâmetro $\mu$, a cada iteração, é multiplicado por uma constante $\sigma$ positiva $(\sigma \leq 1)$ (Martinez, 1998). Isto faz com que o valor de $(1 / 2 \mu)$ aumente com as iterações, penalizando ainda mais as violações das restrições do problema. No tocante aos $\lambda_{m}$, existem várias formas de se efetuar as suas atualizações, sendo o método de Multiplicadores de Restrições de Igualdade (Martinez, 1998) largamente utilizado na literatura, devido a sua simplicidade. Neste caso, as atualizações dos multiplicadores de Lagrange são realizadas levando em conta a magnitude dos desvios das restrições $c_{m}(x)$, ou seja:

$$
\lambda_{m}^{i t+1}=\lambda_{m}^{i t}-c_{m}\left(x^{i t}\right) / \mu^{i t}(5.2 .3)
$$

A convergência desse algoritmo é alcançada quando uma determinada solução for factível e o gradiente do LA, $\nabla \ell_{A}$, possuir norma menor que uma determinada tolerância:

$$
\nabla \ell_{A}=\nabla f(x)+\sum_{m=1}^{M}\left[\lambda_{m}-\frac{c_{m}(x)}{\mu}\right] \nabla c_{m}(x)
$$

Nessa situação, as condições de otimalidade de primeira ordem são atendidas, ou seja, é possível descrever o gradiente da função objetivo do problema original, $\nabla f(x)$, como combinação convexa dos gradientes das restrições, $\nabla c_{m}(x)$.

Para aplicar o LA ao Problema (5.1.12), primeiramente é necessário transformar suas restrições em igualdades. Para tanto, são utilizadas algumas variáveis de folga. Assim, para reservatório $r$ e estágio $t$, o subproblema a ser resolvido é dado por:

$$
\begin{array}{r}
\Theta_{D U H}(.)=\min -\lambda d\left(\sum_{j=1}^{n g} p h_{j}\left(q_{j}, Q, s\right)\right)+ \\
\lambda q \cdot Q+\lambda s \cdot s
\end{array}
$$

$$
\begin{aligned}
& \sum_{j=1}^{n g} q_{j}-Q=0 \\
& p h_{j}\left(q_{j}, Q, s\right)-\beta_{j}=0 j=1, n g \\
& \sum_{j=1}^{n g}\left[p h_{j 1}^{\text {max }}-p h_{j}\left(q_{j}, Q, s\right)\right]-r h-\beta_{R}=0 \\
& p h_{j}^{\text {min }} \leq \beta_{j} \leq p h_{j}^{\text {max }} \\
& \beta_{R}>0
\end{aligned}
$$

Pode-se perceber que o problema possui $2 n g+3$ variáveis e $n g+2$ restrições.

O próximo passo é construir a função LA do Problema (5.2.5):

$$
\begin{aligned}
& \ell_{A}=-\lambda d\left(\sum_{j=1}^{n g} p h_{j}\left(q_{j}, Q, s\right)\right)+\lambda q \cdot Q+\lambda s \cdot s \\
& +\lambda_{1}\left(\sum_{j=1}^{n g} q_{j}-Q\right)+\sum_{j=1}^{n g} \lambda_{j+1}\left(p h_{j}\left(q_{j}, Q, s\right)-\beta_{j}\right) \\
& +\lambda_{m}\left(\sum_{j=1}^{n g}\left(p h_{j 1}^{\max }-p h_{j}\left(q_{j}, Q, s\right)\right)-r h-\beta_{R}\right) \\
& +\frac{1}{2 \mu}\left[\left(\sum_{j=1}^{n g} q_{j}-Q\right)^{2}+\sum_{j=1}^{n g}\left(p h_{j}\left(q_{j}, Q, s\right)-\beta_{j}\right)^{2}\right] \\
& +\frac{1}{2 \mu}\left(\sum_{j=1}^{n g}\left[p h_{j 1}^{\max }-p h_{j}\left(q_{j}, Q, s\right)\right]-r h-\beta_{R}\right)^{2}
\end{aligned}
$$

\section{APLICAÇÃO}

Para aplicar a metodologia proposta aos problemas de alocação e despacho de unidades hidrelétricas ${ }^{4}$ são utilizados dados das usinas de Ilha Solteira e Salto Osório. A aplicação considerou Ilha Solteira com 18 unidades, cada qual com uma zona permitida de operação definida pelo intervalo [60,165] MW. Por sua vez, Salto Osório apresenta 6 unidades divididas em dois grupos: o primeiro (Tipo I) é formado por 4 unidades, cada qual com uma zona operativa definida pelo intervalo $[120,182] \mathrm{MW}$ e o segundo (Tipo II), com 2 unidades, cada qual com sua zona permitida entre os limites $[120,175]$ MW.

De acordo com (5.1.11), é necessário definir os multiplicadores vindos do Problema Dual (5.3), ou seja, $\lambda d_{r t}, \lambda q_{r t}$ e $\lambda s_{r t}$. Esses valores, que são provenientes de uma determinada iteração da solução do Problema (5.3), estão mostrados na Tabela 6.1.

\footnotetext{
${ }^{4}$ Os subproblemas de Alocação e Despacho de Unidades Hidrelétricas são resolvidos para cada usina $r$ e estágio de tempo t. Suas soluções independem dos acoplamentos espaciais existentes, ou seja, da presença de usinas à montante ou jusante.
} 


\begin{tabular}{lccc}
\hline & $\lambda \boldsymbol{d}_{r t}$ & $\lambda \boldsymbol{q}_{r t}$ & $\lambda \boldsymbol{s}_{r t}$ \\
\hline Ilha Solteira & 5,5 & 2,0 & 1,0 \\
Salto Osório & 3,0 & 2,0 & 1,0 \\
\hline
\end{tabular}

Tabela 6.1: Multiplicadores do Problema Dual.

Além desses multiplicadores, é necessário também definir qual a combinação de unidades (estado) que deve ser utilizada nesse problema, ou seja, quais unidades estarão operando e em qual zona operativa. Essa combinação é informada pelo problema de alocação (5.1.10), onde, a cada iteração, determina uma combinação a ser resolvida pelo problema de despacho.

Inicialmente, a título de ilustração, faz-se uma análise do algoritmo para as combinações que utilizam o número máximo de unidades por usinas (Ilha Solteira com 18 unidades e Salto Osório com seis unidades).

A Tabela 6.2 ilustra os principais resultados obtidos ao longo do processo iterativo:

\begin{tabular}{ccc}
\hline & Ilha Solteira & Salto Osório \\
\hline Tempo Computacional & $1,9 \mathrm{~s}$ & $0,3 \mathrm{~s}$ \\
Número de Iterações & 44 & 14 \\
$\left\|c_{m}(x)\right\|_{\infty}$ & $6,7 \times 10^{-4}$ & $1,0 \times 10^{-3}$ \\
$\left\|\nabla \ell_{A}\right\|_{\infty}$ & $5,0 \times 10^{-3}$ & $1,0 \times 10^{-8}$ \\
\hline
\end{tabular}

Tabela 6.2: Desempenho Computacional.

onde $\left\|c_{m}(x)\right\|_{\infty}$ e $\left\|\nabla \ell_{A}\right\|_{\infty}$ correspondem às normas dos desvios das restrições e do gradiente do LA, respectivamente.

O problema de despacho de Salto Osório possui 15 variáveis e 8 restrições, enquanto que, Ilha Solteira é formado por 39 variáveis e 20 restrições, justificando o maior o esforço computacional apresentada por esta última.

Na sequiência, as Figuras 6.1 e 6.2 mostram a evolução das normas dos desvios das restrições e do gradiente do LA ao longo do processo iterativo.

Pode-se observar nessas figuras que, inicialmente, há as maiores violações das restrições. À medida que o processo iterativo evolui, o gradiente do LA aproxima-se de zero e as soluções factíveis são encontradas.

Com relação à solução do problema de despacho de unidades hidrelétricas, para ambos os casos, as figuras 6.3, 6.4 e 6.5 mostram os valores da vazão turbinada e potência de saída para cada unidade.

Para o caso de Salto Osório, percebe-se que a solução mostra

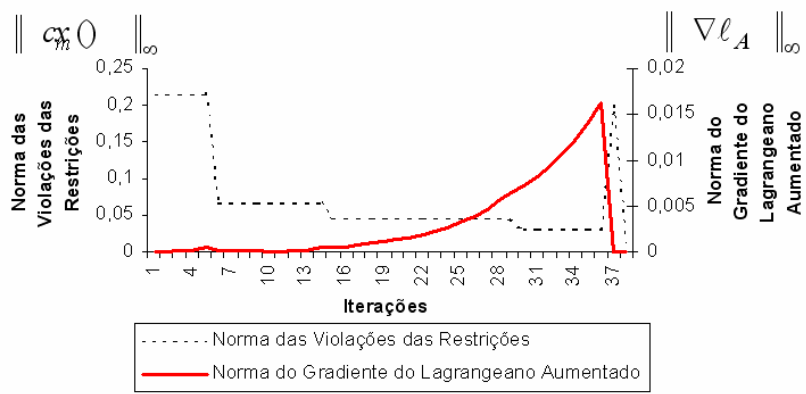

Figura 6.1: Ilha Solteira - Convergência.

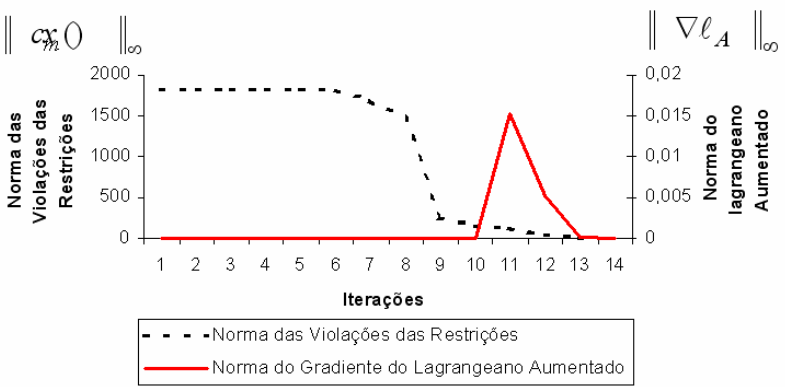

Figura 6.2: Salto Osório - Convergência.

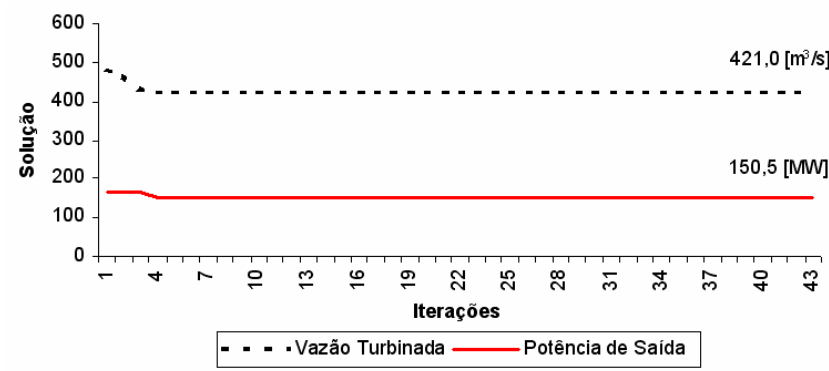

Figura 6.3: Ilha Solteira - Solução.

despachos diferentes para as unidades dos Tipos I e II. Na Figura 6.4 o despacho encontrado foi de 146,9 MW enquanto para a Figura 6.5 mostra 165,2 MW. Esta diferença é justificada pelo fato de que as curvas-colina das unidades serem diferentes.

Para completar o estudo, na sequiência mostra-se a aplicação do algoritmo para a solução final do problema de alocação de unidades, resolvendo-se as outras combinações possíveis do despacho, isto é, a enumeração exaustiva de todo o espaço de estados do problema. Para tanto, é necessário resolver, para cada estado, um problema de despacho de unidades geradoras hidrelétricas (5.1.11). Para os casos considerados como exemplo, as enumerações exaustivas de Ilha Solteira e Salto 


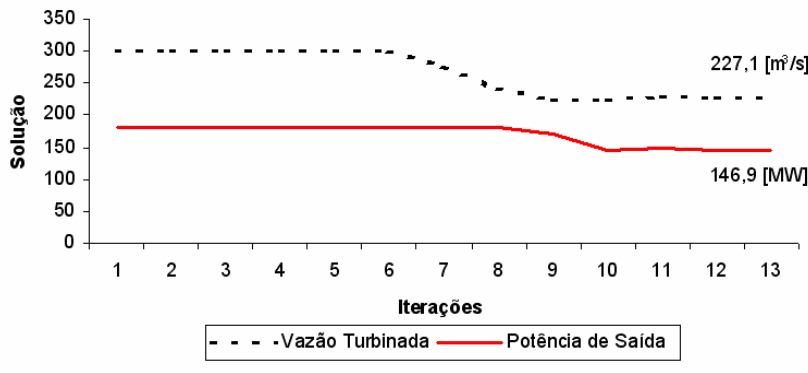

Figura 6.4: Salto Osório - Solução - Unidades Tipo I.

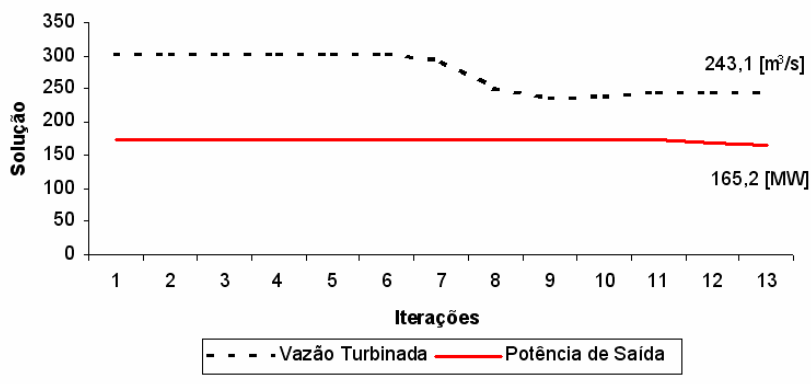

Figura 6.5: Salto Osório - Solução - Unidades Tipo II.

Osório estão mostradas na Figura 6.6 e na Tabela 6.3.

Para Ilha Solteira, pode-se observar o comportamento nãolinear do gráfico. Considerando os preços da Tabela 5.3.1, a melhor combinação de despacho requer 11 unidades em operação, com o valor da função objetivo aumentando consideravelmente para as demais combinações.

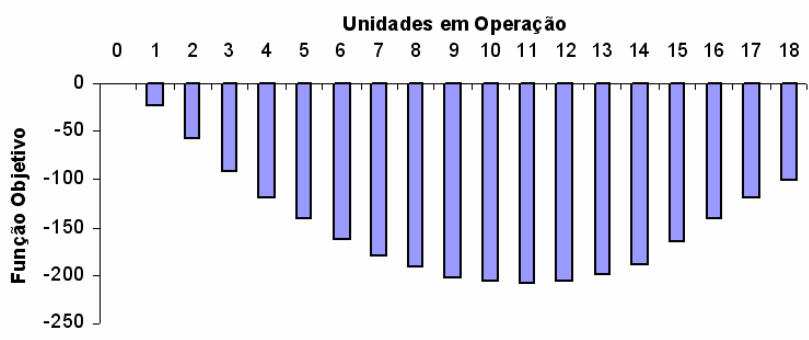

Figura 6.6: Ilha Solteira - Enumeração Exaustiva.

Para o caso de Salto Osório, pode-se observar o menor valor da função objetivo é obtido não despachando nenhuma unidade dessa usina.

Contudo, aumentando-se o valor do preço da potência para $\lambda d_{r t}=3,15$, uma nova alocação ótima é obtida, conforme apresentado na Tabela 6.4.

Com base nos resultados mostrados acima, pode-se notar

\begin{tabular}{lr|ccccc}
\hline \multicolumn{2}{c|}{ Valores da } & \multicolumn{5}{c}{ Unidades do Tipo I } \\
\multicolumn{2}{c|}{ Função Objetivo } & $\mathbf{0}$ & $\mathbf{1}$ & $\mathbf{2}$ & $\mathbf{3}$ & $\mathbf{4}$ \\
\hline \multirow{2}{*}{ Unidades } & $\mathbf{0}$ & $\mathbf{0 , 0}$ & 3,70 & 3,48 & 14,55 & 31,08 \\
do Tipo II & $\mathbf{1}$ & 5,26 & 1,10 & 11,79 & 28,25 & 50,24 \\
& $\mathbf{2}$ & 0,57 & 7,93 & 25,45 & 48,26 & 74,92 \\
\hline
\end{tabular}

Tabela 6.3: Salto Osório - Enumeração Exaustiva.

\begin{tabular}{lr|ccccc}
\hline \multicolumn{2}{c|}{ Valores da } & \multicolumn{5}{c}{ Unidades do Tipo I } \\
\multicolumn{2}{l|}{ Função Objetivo } & $\mathbf{0}$ & $\mathbf{1}$ & $\mathbf{2}$ & $\mathbf{3}$ & $\mathbf{4}$ \\
\hline \multirow{2}{*}{ Unidades } & $\mathbf{0}$ & 0,0 & $-17,3$ & $-44,8$ & $-57,9$ & $-63,5$ \\
\multirow{2}{*}{ do Tipo II } & $\mathbf{1}$ & $-14,7$ & $-46,2$ & $-62,7$ & $-67,1$ & $-67,2$ \\
& $\mathbf{2}$ & $-45,6$ & $-65,6$ & $\mathbf{- 7 2 , 4}$ & $-70,1$ & $-64,2$ \\
\hline
\end{tabular}

Tabela 6.4: Salto Osório $-\lambda \boldsymbol{d}_{r t}=\mathbf{3 , 1 5}$.

que, uma pequena variação no multiplicador $\lambda d_{r t}$ proporcionou uma grande mudança na alocação dessas unidades. A solução aqui encontrada para a alocação determina a operação com duas unidades do Tipo I e duas do Tipo II, com valor da função objetivo, para este estado, de $-72,4$.

\section{CONCLUSÕES}

Neste artigo foi abordado o problema de programação da operação energética de sistemas hidrotérmicos. Esse problema é de difícil solução por ser não-linear, inteiro-misto e de grande porte. A técnica de Relaxação Lagrangeana foi utilizada para decompor o problema em subproblemas menores, entre eles o subproblema de despacho das unidades geradoras hidrelétricas, de natureza não-linear, mas contínuo. Um método de solução baseado no Lagrangeano Aumentado foi implementado para a solução desse subproblema. Os detalhes do método e os resultados dessa aplicação são apresentados.

Conforme visto, os resultados obtidos mostram o efeito da modelagem detalhada das unidades hidrelétricas, onde, os níveis de geração dependem da vazão turbinada, vertimento e altura de queda líquida. Também pode ser observado que os despachos das unidades geradoras são dependentes dos multiplicadores da função dual $\left(\lambda d_{r t}, \lambda q_{r t}\right.$ e $\left.\lambda s_{r t}\right)$ e, também, do número (combinação) de unidades em operação. Investigando todo o espaço de estados por meio de uma enumeração exaustiva obteve-se a alocação ótima das unidades.

A aplicação realizada mostrou que o método de LA é eficaz na solução do problema de despachos de unidades geradoras, o que lhe qualifica como promissor para implementações práticas. 


\section{REFERÊNCIAS}

Batut, J., Renaud, A. (1992). Daily Generation Scheduling with Transmission Constraints: A New Class of Algorithms, IEEE Transactions on Power Systems, Volume 7, Number 3.

Bellman, R. (1957). Dynamic Programming, Princeton, N. J., Princeton University Press.

Bertsekas, D. P. (1999); Nonlinear Programming, Athena Scientific, Second Edition.

Bonnans, J. F., Gilbert, J. C., Lemaréchal, C. Sagastizábal, C. (2002); Numerical Optimization. Theorical and Pratical Aspects, universitext, Springer-Verlag, Berlin.

Cicogna, M., Arce, A. E., Soares Filho, S., Ohihi, T. (2003); Dynamic Dispatch of Hydro Generation Units, IEEE bologna Power Tech Conference, Bologna.

Douglas, J., Rachford, H. H, (1956); On The Numerical Solution of Heat Conduction Problems in Two and Three Variables, Trans. Maer. Math. Soc., 82:421-439.

Finardi, E. C. ; Silva, E. L.; Sagástizabal, C. A. (2005); Solving the Unit Commitment Problem of Hydropower Plants Via Relaxation Lagrangian and Sequential Quadratic Programming. Matematica aplicada e computacional, Brasil, v. 24, n. 3, p. 1-25,.

Geoffrion, A. M. (1971). Duality in Nonlinear Programming: A Simplified Applications-Oriented Development, SIAM Review, January, Vol. 13, Number 4.

Lasdon, L. S. (1970). Optimization Theory of Large Systems, Macmillan Company, New York, NY.

Lemarechal, C., Pellegrino, F., Renaud, A., Sagastizabal, C. (1996). Bundle Methods Applied to the Unit Commitment Problem, System Modeling and Optimization, pp. 395-402.

Martínez, J. M. (2000). Practical Quasi-Newton Methods for Solving Nonlinear Systems, Journal of Computational and Applied Mathematics, 124, pp. 97-122.

Martínez, J. M., Santos, S. A. (1998). Métodos Númericos de Otimização, IMECC-UNICAMP,.

Martínez, J. M., Mello, M., Pilotta, E. A., Krejic, N. (2000). Validation of na Augmented Lagrangian Algorithm with a Gauss-Newton Hessian Aproximation Using a Set of Hard-Spheres Problems, Computational Optimization and Applications 16, 247-263.

Merlin, A., Sandrin, P. (1983). A New Method for Unit Commitment at Électricité de France, IEEE Transactions on Power Apparatus and Systems, Volume PAS-102, Number 5 .
Nocedal, J., Wright, S. J. (1999). Numerical Optimization, Springer Series in Operations Research.

Renaud, A. (1993). Daily Generation Management at Életricité de France: From Planning Towards Real Time, IEEE Transactions on Automatic Control, 38(7)1080:1093.

Ruszczynski, A. (1997); Decomposition Methods in Stochastic Programming, Mathematical Programming, 79:333-353.

Silva, E. L. (2001). Formação de Preços em Mercados de Energia Elétrica, Editora Sagra Luzzatto.

Silva, E. L., Finardi, E. C. (2003); Parallel Processing Applied to The Planning of Hydrothermal Systems, IEEE Transactions on Parallel and Distributed Systems, Volume 14, August.

Urruty, J. B. H., Lemarechal, C. (1993). Convex Analysis and Minimization Algorithms, Springer-Verlag,.

Wonnacott, T. H., Wonnacott, R. J. (1972); Introductory Statistics of Businness and Economics, Ontaro, Canada, John Wiley \& Sons, Inc.

Wood, A., Wollenberg, B. (1984); Power Generation Operation \& Control, John Wile \& Sons.

Zhuang, F., Galiana, F. D. (1988). Towards a More Rigorous and Practical Unit Commitment by Lagrangian Relaxation, IEEE Transactions on Power Systems, Volume 3, Number 2. 